Original Article

\title{
One-year results of voluntary-based supervised exercise or treatment at orthopedic clinic for radiographic severe knee osteoarthritis
}

\author{
Hirofumi Miyagawa, $\mathrm{PT}^{1)}$, Tatsunori Ikemoto, $\mathrm{MD}^{1)^{*}}$, Hiroshi Honjo, $\mathrm{MD}^{2)}$, \\ Machiko AKaO, $\mathrm{MD}^{2)}$, ToshiYa Tsujimoto, $\mathrm{MD}^{2)}$, TAKahiro Ushida, $\mathrm{MD}^{1)}$ \\ 1) Institute of Physical Fitness, Sports Medicine and Rehabilitation, Aichi Medical University: \\ 1-1 yazako karimata, Nagakute, Aichi 480-1195, Japan \\ 2) Department of Orthopedic Surgery, Aichi Medical University, Japan
}

\begin{abstract}
Purpose] In this study, we investigated the efficacy of supervised physical exercise or conventional treatment on symptomatic knee osteoarthritis with severe morphological degeneration. [Subjects] Sixty-six patients with severe radiographic knee osteoarthritis were enrolled. [Methods] Participants were separated into two groups: in one group patients conducted physical exercise under supervision; while in the other group they were treated by conventional clinical methods for one year. Participants filled out two types of questionnaires; the Japanese Knee Osteoarthritis Measure and the Pain Disability Assessment Scale at baseline and one year following enrollment in the study. Two-way repeated measures analysis of variance was used to examine the effects over time and by group for a total of 43 participants; consisting of an exercise group $(n=20)$ and a clinical group $(n=23)$ excluding 23 dropouts. [Results] Analysis did not show a significant time-course effect or interaction between time-course and the groups in both questionnaires. On the other hand, there were significant group effects in both questionnaires with an advantage in the exercise group. [Conclusion] These results indicate that patients with knee osteoarthritis under supervised exercise conditions are more likely to maintain a better clinical outcome at one-year follow-up, despite the severe morphological degeneration in their knees.

Key words: Knee osteoarthritis, Physical exercise
\end{abstract}

(This article was submitted Oct. 21, 2015, and was accepted Dec. 12, 2015)

\section{INTRODUCTION}

Osteoarthritis (OA) of the knee is a common problem causing knee pain and physical disabilities in the elderly ${ }^{1)}$. The prevalence of knee OA is known to increase with age not only in Japan but also in various countries, meaning it is an important social issue around the world ${ }^{2}$, 3). Recently, Research on Osteoarthritis Against Disability (ROAD) studies in Japan revealed an association between knee pain and joint space narrowing in men, while women tended to have knee pain even without radiographic knee OA. In general, a progression of radiographic change is thought to be a crucial indicator for assessing the severity of knee $\mathrm{OA}^{4,5)}$. However, there is a widespread belief of inconsistency between clinical symptoms and radiographic severity 6,7 .

There are various factors associated with the symptoms of knee OA. For instance, Sun et al. reported that the serum IL-15 levels independently and positively correlated with pain severity but not KL grades in OA patients and, therefore, joint inflammation might play a key role, reflected by the severity of the symptoms ${ }^{8}$.

The OA Research Society International (OARSI) guidelines for management of knee OA established by several orthopedic associations in each country ${ }^{9}$, recommend physical exercises as a core treatment method. Indeed, some articles revealed

\footnotetext{
*Corresponding author. Tatsunori Ikemoto (E-mail: tatsunon31-ik@umin.ac.jp)

(C)2016 The Society of Physical Therapy Science. Published by IPEC Inc.

This is an open-access article distributed under the terms of the Creative Commons Attribution Non-Commercial No Derivatives (by-nc-nd) License $<$ http://creativecommons.org/licenses/by-nc-nd/4.0/>.
} 
that aerobic exercise and resistance training decreased serum levels of inflammatory mediators ${ }^{10)}$.

Also, the Multicenter Osteoarthritis study recently reported a significant concordance between knee pain and radiographic knee OA by Kellgren and Lawrence grades ${ }^{11}$. Although this study implies a decreased efficacy of physical exercises for progressive knee OA, there is little evidence indicating the effectiveness of physical exercise in cases of severe radiographic knee OA.

Therefore, in this study, we tried to investigate the efficacy of supervised physical exercise for the symptoms in patients with knee OA by separating them into two groups: one group consisting of patients conducting supervised physical exercise; and the other treated by conventional clinical methods. For our results, we used both the Japanese Knee Osteoarthritis Measure $(\mathrm{JKOM})^{12}$ and the Pain Disability Assessment Scale (PDAS) ${ }^{13}$. The JKOM has been established to assess the activity of daily life as well as pain and stiffness deriving from patients with knee OA in Japan. Furthermore, we also evaluated the pain related disabilities in patients with severe knee OA using the PDAS because previous reports indicated that knee pain was subject to vary within months and weeks irrespective of radiographic severity ${ }^{14}$.

This prospective study was conducted longitudinally for one year to confirm the mid-term efficacy of each treatment method.

\section{SUBJECTS AND METHODS}

The study received ethical approval from the Research Ethics Committee of the Aichi Medical University (No.12-101).

Patients with knee pain, confirmed with radiographic knee OA with a face of grade 3 or above according to the KellgrenLawrence (K-L) grading system were eligible for this study. Eligibility criteria included patients older than 50 years and without total knee arthroplasty in either knee. We recruited participants who were interested in this study, which investigated knee-related health conditions using a specific questionnaire. We enrolled sixty-six subjects into this study. We divided subjects into one of two different groups based on their knee care. We defined subjects in one group ( $\mathrm{n}=26$ ) as doing exercise for at least one year or more at a physical fitness center in Aichi Medical University regardless of whether they were receiving other forms of treatment or not (exercise group). We defined subjects in the other group ( $\mathrm{n}=40)$ as treated at an orthopedic clinic in Aichi Medical University without using any physical fitness center (clinical group). Accordingly, what treatment subjects received was already decided, and, therefore, it was hard to change the arm. As a result, this study was a non-randomized clinical trial.

Before investigating their status, each subject was fully informed by an investigator: 1) on the content of this study and 2) that all personal information of the subject would be kept confidential.

After investigating their demographic backgrounds (age, gender), subjects received a radiographic examination of both knees by the posterior-anterior view at standing position to confirm radiographic severity. These radiographs were assessed by at least two orthopedic physicians according to the K-L grading system that uses the following grades: 0 , normal; 1 , possible osteophytes only; 2, definite osteophytes and possible joint space narrowing; 3, moderate osteophytes and/or definite joint space narrowing; and 4, large osteophytes, severe joint space narrowing, and/or bony sclerosis ${ }^{6}$. Substantially, each radiographic score was decided according to the higher K-L grade of either side.

Participants also filled out two types of questionnaires, the Japanese Knee Osteoarthritis Measure (JKOM) ${ }^{12)}$ and the Pain Disability Assessment Scale (PDAS) to assess their knee pain and pain-related disabilities ${ }^{13)}$. The JKOM consists of a pain rating based on a 100-mm visual analog scale (VAS) and scores for a subscale of four symptoms based on a diseasespecific questionnaire addressing four dimensions: 'pain/stiffness', 'condition in daily life', 'general conditions' and 'health conditions', with 8, 10, 5 and 2 questions, respectively ${ }^{12}$. Questions are rated on an ordinal scale of $0-4$, with higher scores indicating a symptom or medical condition of higher severity. The four symptom subscales can be scored separately or combined to represent the aggregated total symptoms. On the other hand, the PDAS is a scale for measuring lifestyle disabilities of patients with chronic pain. Higher scores (on a scale of 0 to 60 points) indicate greater degrees of lifestyle disability ${ }^{13)}$.

After one-year follow-up, three patients in the exercise group and 17 patients in the clinical group were excluded as they had dropped out of the study. Reasons for dropout were different for each participant and included receiving total knee arthroplasty (one subject), seeking other treatments, and unknown reasons. Accordingly, we collected data for this research study from 43 patients in total, of which 20 patients were from the exercise group and 23 patients were from the clinical group

Voluntary-based supervised exercise consisted of a complex program such as land-based aerobic activities ${ }^{15)}$ (e.g. cycling), stretching ${ }^{16)}$, muscle strengthening (e.g. quadriceps and hamstrings strengthening) ${ }^{17)}$ and aqua-based exercise (e.g. water walking $)^{18)}$ in our fitness center. We adjusted these exercise programs for each individual under the supervision of a physical therapist. For instance, subjects were instructed on knee stretching based on our stretching technique that could stretch the target muscle without decreasing muscle strength ${ }^{19}$. Subjects used muscle strengthening exercises with some machines for both quadriceps and hamstrings, however when the subject complained of knee pain during the exercise, the magnitude of leg load was regulated according to the severity of the symptoms to prevent an aggravation of symptoms or subjects were instructed to only isometric quadriceps contraction ${ }^{20)}$. Moreover, when the subject had knee swelling, only water walking was advised as aerobic exercise by a therapist without cycling or treadmill walking. An exercise session was approximately 90 to 120 minutes divided by several breaks for each subject adjusted by endurance. Although the frequency of exercise was different for each subject according to their convenience, everyone exercised one to three times in a week but 
at least once a week in our fitness center. Seven participants in the exercise group regularly received intra-articular hyaluronic acid injections in other clinics once a month.

By contrast, conventional treatment in the clinic included nonsteroidal anti-inflammatory drugs (NSAIDs) and intraarticular hyaluronic acid injections. Patient consultations were usually conducted once or twice a month for each subject.

At baseline, we compared demographic and morphological parameters among the groups, for age using the MannWhitney $\mathrm{U}$ test and for gender and K-L grades using the $\chi^{2}$ test. Two-way repeated measures analysis of variance (ANOVA) was performed to examine the effects over time and by group on the JKOM score and PDAS score with a Scheffé post-hoc comparison. Subsequently, four symptom subscales of JKOM were evaluated to examine separate effects of each treatment using the Wilcoxon signed-rank test. We preformed all analyses with SPSS software (version $20.0 \mathrm{~J}$; SPSS Inc., Chicago, IL, USA). We considered $\mathrm{p}<0.05$ as statistically significant.

\section{RESULTS}

There was no significant difference in the epidemiological background (age $\mathrm{p}=0.57$, gender ratio $\mathrm{p}=0.54$ ) and radiographic severity of knees among the two groups, although there was a different trend in the severity of K-L grade ( $\mathrm{p}=0.07)($ Table 1$)$.

Two-way repeated measures ANOVA did not show a significant time-course effect $(\mathrm{F}=0.04 \mathrm{p}=0.83)$ or interaction $(\mathrm{F}=3.40$ $\mathrm{p}=0.07)$ between time-course and group in JKOM score. Similarly, it did not show any significant time-course ( $\mathrm{F}=0.27$ $\mathrm{p}=0.61)$ or interaction $(\mathrm{F}=0.17 \mathrm{p}=0.69)$ between time-course and group in the PDAS score. However, there were significant group effects in both the JKOM score $(\mathrm{F}=6.98 \mathrm{p}=0.01)$ and PDAS score $(\mathrm{F}=5.49 \mathrm{p}=0.02)$.

Post hoc analysis revealed that only the patients in the exercise group showed significant improvement in their JKOM score at one year after baseline ( $\mathrm{p}<0.05$, Scheffé comparison). Moreover, they revealed a significant difference in PDAS score among the groups at baseline $(\mathrm{p}<0.05)$, although there is no significant difference in JKOM score (Table 2). On the other hand, in the JKOM subscale, the scores related to general conditions and health conditions in the exercise group had significantly improved at one year after baseline ( $p<0.01$, Wilcoxon signed-rank test). In contrast, the score related to pain/ stiffness in the clinical group had significantly declined one year after baseline ( $p<0.05$, Wilcoxon signed-rank test) (Table 3 ).

Table 1. Patients' demographic background

\begin{tabular}{lcc}
\hline & Exercise group & Clinical group \\
\hline Age (years) & $72.0 \pm 6.1$ & $73.9 \pm 6.9$ \\
Male / Female & $5 / 15$ & $4 / 19$ \\
Grade 3 / Grade 4 & $15 / 5$ & $11 / 12$ \\
\hline
\end{tabular}

Ages are reported as mean $\pm \mathrm{SD}$.

Each category was compared among the groups, for age using the Mann-Whitney U test and for gender and K-L grades using $\chi^{2}$ test. Both analyses showed no significant difference among the groups $(\mathrm{p}>0.05)$.
Table 2. JKOM score and PDAS score at baseline and oneyear later in each group

\begin{tabular}{lcc}
\hline & Baseline & One-year later \\
\hline JKOM & & \\
Exercise group & $20.8 \pm 16.0$ & $17.3 \pm 14.4^{\$ * \#}$ \\
Clinical group & $27.0 \pm 15.5$ & $29.8 \pm 15.1$ \\
PDAS & & \\
Exercise group & $11.4 \pm 9.4^{\#}$ & $9.1 \pm 11.4^{\$ * \# \#}$ \\
Clinical group & $16.4 \pm 10.8$ & $16.3 \pm 10.4$ \\
\hline
\end{tabular}

Data are reported as mean \pm SD.

${ }^{\$} \mathrm{p}<0.05$, Within group difference. (Two-way repeated measures ANOVA)

${ }^{*} \mathrm{p}<0.05$, Baseline vs. One-year later; ${ }^{\#} \mathrm{p}<0.05,{ }^{\#} \mathrm{p}<0.01$ Exercise vs. Clinical (Post hoc Scheffé comparison)

JKOM: Japanese Knee Osteoarthritis Measure, PDAS: Pain Disability Assessment Scale

Table 3. Outcome on subscales of JKOM score in each group

\begin{tabular}{lcccc}
\hline & \multicolumn{2}{c}{ Exercise group } & \multicolumn{2}{c}{ Clinical group } \\
\cline { 2 - 5 } & Baseline & One-year later & Baseline & One-year later \\
\hline Pain/Stiffness & $8.3 \pm 6.2$ & $6.2 \pm 4.9$ & $9.6 \pm 5.6$ & $11.5 \pm 5.8^{*}$ \\
Condition in daily life & $6.2 \pm 5.3$ & $4.2 \pm 5.3$ & $9.7 \pm 6.7$ & $10.4 \pm 6.7$ \\
General conditions & $3.3 \pm 4.5$ & $1.1 \pm 2.8 * *$ & $4.9 \pm 4.6$ & $4.7 \pm 4.0$ \\
Health conditions & $3.0 \pm 1.6$ & $1.9 \pm 1.3 * *$ & $2.9 \pm 1.4$ & $3.1 \pm 1.2$ \\
\hline
\end{tabular}

Data are reported as mean \pm SD.

${ }^{*} \mathrm{p}<0.05,{ }^{* *} \mathrm{p}<0.01$, Baseline vs. One-year later (Wilcoxon signed-rank test)

JKOM: Japanese Knee Osteoarthritis Measure 


\section{DISCUSSION}

What is novel about this study is how we revealed that patients with knee OA conducting supervised exercise have the potential to maintain better clinical outcomes at one-year follow-up, despite severe morphological degeneration in their knees. Physical exercise is recommended ${ }^{9}$ as a core treatment for knee OA. However, evidence on the efficacy of physical exercise for severe knee OA is limited to short-term studies as previous studies were short-term; usually conducted as a patient waited for total knee joint replacement ${ }^{21)}$.

The consensus on the non-surgical management of knee OA includes stepwise treatment options such as voluntary-based physical exercise, education for weight control, physiotherapy, analgesic drugs such as NSAIDs and acetaminophen, and intra-articular injection of steroids and hyaluronic acids ${ }^{22}$. In cases where this fails, total joint replacement with follow-up rehabilitation should be conducted. On the other hand, the present study showed that there was a significant difference between the exercise group and the clinical group regarding the clinical outcomes at one-year follow-up with an advantage in the exercise group. This result indicates that treatment methods for severe knee OA may be an important factor preventing knee arthroplasty in the future, although several studies have indicated that the proportion of knees with a KL grade of 3 or 4 exceeded $95 \%$ in patients scheduled for knee joint replacement ${ }^{23,24)}$.

Our study also documented that supervised physical exercise particularly improved general conditions and health conditions but not the pain/stiffness condition in the JKOM score. We believe that the former finding offers useful insight into patients with severe radiographic knee OA to prolong the life of native knees regardless of pain and functional decline, although these effects might derive from not only an exercise but also a self-care motivation. However, the latter result and decline in the pain-subscale in the clinical group may be associated with previous descriptions ${ }^{7,11)}$. Moreover, although our exercise program included land-based aerobic activities, muscle strength, and aqua-based exercises, based on much evidence in the literature ${ }^{9)}$, we found that it had limited efficacy on pain in knees suffering from severe degeneration. This may indicate that chronic morphological degeneration may have the effect of decreasing the regenerative capacity in local tissues ${ }^{24)}$.

We have to consider several limitations of this study. First, the present study was a non-randomized trial. In particular, the motivational component for one's health such as the ability of self-efficacy was not excluded, although self-efficacy is an important factor for keeping in sound condition even if people have chronic pain. In our study, the participants in the exercise group were probably highly motivated to take care of themselves. In contrast, although the subjects in the clinical group were instructed to follow voluntary home exercises in accordance with their physician's guidelines, we speculate that most did not carry them out with a passive treatable attitude. We could not determine the causes of participant dropout, but we speculate that a difference in the dropout rate between the exercise group (13\%) and the clinical group (46\%) derived from the difference in their self-care motivation. Certainly, there was a significant difference in PDAS scores among the groups at the beginning of this study. Hence, we think that the motivational component has influenced the results of this study. Secondly, we saw many patients in the clinical group drop out of the study. As several studies have reported elsewhere, evidence on the effectiveness of non-pharmacological and non-surgical approaches for severe knee OA are limited to the short-term. In contrast, longitudinal studies are scarce. We think this is because knee arthroplasty can lead to an early solution to knee problems ${ }^{9)}$. Therefore long-term studies in severe knee OA are difficult. Excluding cases in which the patient wants to keep their native knees and self-care motivation bias will exist as long as we examine the long-term effects of physical exercise for severe knee OA. Moreover, most of the patients with severe knee OA are elderly people, and they usually have many disorders, not only in the knee. Hence, general problems among elderly patients might be the reason they drop out of longitudinal studies. Thirdly, although there was no statistical difference between the exercise group and the clinical group regarding their radiological severities, the rate of mild degeneration (grade 3>grade 4) was higher in the exercise group than in the clinical group. Comparative analysis between the groups at baseline showed that this might affect the main results of this study. Therefore, we believe further studies need to be conducted to confirm the effect of supervised exercise on severe knee OA by investigating both the survival rate of native knees and the life expectancy in such patients.

\section{REFERENCES}

1) Sharma L, Kapoor D, Issa S: Epidemiology of osteoarthritis: an update. Curr Opin Rheumatol, 2006, 18: $147-156$. [Medline] [CrossRef]

2) Muraki S, Oka H, Akune T, et al.: Prevalence of radiographic knee osteoarthritis and its association with knee pain in the elderly of Japanese population-based cohorts: the ROAD study. Osteoarthritis Cartilage, 2009, 17: 1137-1143. [Medline] [CrossRef]

3) Urwin M, Symmons D, Allison T, et al.: Estimating the burden of musculoskeletal disorders in the community: the comparative prevalence of symptoms at different anatomical sites, and the relation to social deprivation. Ann Rheum Dis, 1998, 57: 649-655. [Medline] [CrossRef] 
4) Kellgren JH, Lawrence JS: Radiological assessment of osteo-arthrosis. Ann Rheum Dis, 1957, 16: 494-502. [Medline] [CrossRef]

5) Emrani PS, Katz JN, Kessler CL, et al.: Joint space narrowing and Kellgren-Lawrence progression in knee osteoarthritis: an analytic literature synthesis. Osteoarthritis Cartilage, 2008, 16: 873-882. [Medline] [CrossRef]

6) Claessens AA, Schouten JS, van den Ouweland FA, et al.: Do clinical findings associate with radiographic osteoarthritis of the knee? Ann Rheum Dis, 1990, 49: 771-774. [Medline] [CrossRef]

7) Kocak FU, Unver B, Karatosun V, et al.: Associations between radiographic changes and function, pain, range of motion, muscle strength and knee function score in patients with osteoarthritis of the knee. J Phys Ther Sci, 2009, 21: 93-97. [CrossRef]

8) Sun JM, Sun LZ, Liu J, et al.: Serum interleukin-15 levels are associated with severity of pain in patients with knee osteoarthritis. Dis Markers, 2013, 35: 203-206. [Medline] [CrossRef]

9) McAlindon TE, Bannuru RR, Sullivan MC, et al.: OARSI guidelines for the non-surgical management of knee osteoarthritis. Osteoarthritis Cartilage, 2014, 22: 363-388. [Medline] [CrossRef]

10) Donges CE, Duffield R, Drinkwater EJ: Effects of resistance or aerobic exercise training on interleukin-6, C-reactive protein, and body composition. Med Sci Sports Exerc, 2010, 42: 304-313. [Medline] [CrossRef]

11) Neogi T, Felson D, Niu J, et al.: Association between radiographic features of knee osteoarthritis and pain: results from two cohort studies. BMJ, 2009, 339: b2844. [Medline] [CrossRef]

12) Akai M, Doi T, Fujino K, et al.: An outcome measure for Japanese people with knee osteoarthritis. J Rheumatol, 2005, 32: 1524-1532. [Medline]

13) Yamashiro K, Arimura T, Iwaki R, et al.: A multidimensional measure of pain interference: reliability and validity of the pain disability assessment scale. Clin J Pain, 2011, 27: 338-343. [Medline] [CrossRef]

14) Bellamy N, Sothern RB, Campbell J: Rhythmic variations in pain perception in osteoarthritis of the knee. J Rheumatol, 1990, 17: 364-372. [Medline]

15) Tanaka R, Ozawa J, Kito N, et al.: Effect of the frequency and duration of land-based therapeutic exercise on pain relief for people with knee osteoarthritis: a systematic review and meta-analysis of randomized controlled trials. J Phys Ther Sci, 2014, 26: 969-975. [Medline] [CrossRef]

16) Aoki O, Tsumura N, Kimura A, et al.: Home stretching exercise is effective for improving knee range of motion and gait in patients with knee osteoarthritis. J Phys Ther Sci, 2009, 21: 113-119. [CrossRef]

17) Hafez AR, Al-Johani AH, Zakaria AR, et al.: Treatment of knee osteoarthritis in relation to hamstring and quadriceps strength. J Phys Ther Sci, 2013, 25: 1401-1405. [Medline] [CrossRef]

18) Kim IS, Chung SH, Park YJ, et al.: The effectiveness of an aquarobic exercise program for patients with osteoarthritis. Appl Nurs Res, 2012, 25: 181-189. [Medline] [CrossRef]

19) Inami $T$, Shimizu $T$, Miyagawa $H$, et al.: Influence of various passive triceps surae stretching techiniques on muscle strength. Jpn J Orthop Sports Med, 2009, 29: 168-172.

20) Anwer S, Alghadir A: Effect of isometric quadriceps exercise on muscle strength, pain, and function in patients with knee osteoarthritis: a randomized controlled study. J Phys Ther Sci, 2014, 26: 745-748. [Medline] [CrossRef]

21) Wallis JA, Taylor NF: Pre-operative interventions (non-surgical and non-pharmacological) for patients with hip or knee osteoarthritis awaiting joint replacement surgery - a systematic review and meta-analysis. Osteoarthritis Cartilage, 2011, 19: 1381-1395. [Medline] [CrossRef]

22) Michael JW, Schlüter-Brust KU, Eysel P: The epidemiology, etiology, diagnosis, and treatment of osteoarthritis of the knee. Dtsch Arztebl Int, 2010, 107: 152-162. [Medline]

23) McCusker C, Gardiner DM, Otte KS, et al.: The axolotl model for regeneration and aging research: a mini-review. Gerontology, 2011, 57: 565-571. [Medline] [CrossRef]

24) Richardson JC, Ong BN, Sim J: Experiencing and controlling time in everyday life with chronic widespread pain: a qualitative study. BMC Musculoskelet Disord, 2008, 9: 3. [Medline] [CrossRef] 